Rev. Chil. Pediatr. 66 (2); 83-88, 1995

\title{
Telarquia prematura: Aumento de la actividad estrogénica total en el plasma
}

\author{
Hemán García B. ${ }^{\text {; }}$ Ronald Youlton R. ${ }^{2}$ Luis Valladares B. ${ }^{3}$; Alejandra Jara G. ${ }^{4}$; \\ Andreína Cattani 0.5; Matías Tijmes E. ${ }^{3}$; Cristián García B. ${ }^{5}$
}

\author{
Premature thelarche: evidence of increased \\ serum total estrogenic activity
}

\begin{abstract}
Hormone profile and pelvic ultrasonography were sturbied in 41 girls with premalure thelarche aged $21.2 \pm 9.7$ months ond in 39 non affected con'rol girls of the some age and social stolus. Estrogenic conraminanls were looked tor bolh in girls sero and in poultry somples through serum and tissue levels of zearglenone and diethilbestrol. Total estrogenic activity was measured by radioreceplor assoy. Easal serum levels of LH, FSH, proloctine and esiradiol (E2) were nor different between bath groups. Total estrogenic activity was significantly higher in girls with thelarche $[20] \pm$ 102 vs $78 \pm 20$ pg E2 eovivalent), turning later ro simlar levels than in controls in five patients in which thelarche eventually subsided. After $1 \mathrm{H} R \mathrm{RH}$ grealer FSH than LH peak response was observed $128.7 \pm 17.5 \mathrm{U \mu} / \mathrm{m}$ vs $5 .: \pm 2.7$ $U_{\mu} / \mathrm{m}||$. No differences were delected in uterine size and squeletal maturation, but a greater mean ovarian volume was seen in polienls with thelarquia. No estrogenic conlaminants were detected in poultry samples. Partial activarion of the hypothalamus-pituitaryaxis, mainiy through $\mathrm{FSH}$ secretion, might thus be the responsible of ovarion secretion of E2 ar conother substance with estrogenic action.
\end{abstract}

(Key words: thelarche premature, estrogens, Lti, FSH, releosing hormone, gonadorelin, food contamination.)

La telarquia prematura es el crecimiento uni o bilateral del tejido mamario, de carácter benigno, sin otros signos de desarrollo sexual, que ocurre en niñas entre 6 meses y 8 años de edad ${ }^{1}$. No se conoce exactamente su etiología y los estudios hormonales realizados no han sido concluyentes. En esta comunicación se describen las principales características de laboratorio de un grupo de 41 pacientes con telarquia, y se discuten sus posibles factores etiológicos, en especial la posibilidad de contaminación de algunos alimentos de consumo habitual por niños de esta edad.

1. Hospital Sótero del Río. Instituto de Investigaciones Materno-Infantil. Facultad de Medicina. Lniversidad de Chile.

2. Clínica Las Condes. Departamento de Genética. Facultad de Medicina. Universidad de Chile.

3. Instituto de Nutrición y Tecnología de los Alimentos (INTA).

4. Hospital Roberto del Rio.

5. Hospital Clínico. Facultad de Medicina. Universidad Católica de Chile.

\section{Materiales y métodos}

En un estudio colaborativo se examinaron prospectivamente 41 niñas de $21,15 \pm 9,74$ meses de edad (rango: 6 a 36 meses) que consultaron en las unidades de endocinologia pediátrica de cinco establecimientos de la ciudad de Santiago (Chile), incluyendo los hospizales Sótero del Río (sector sur oriente), Roberto del Río (sector norie), Clínico de la Universidad Católica (sector central) y las clínicas Las Condes y Santa María (sector oriente). por presentar telarquia (aumento de volumen mamario palpa. ble mayor a $2 \mathrm{~cm}$ de diametro, uni o bilateral) prematura. No se adruilicron pacientes que presentaban, además, vello pubiano. aceleración del crecimiento, edad osea adelantada o sangramiento vaginal. En todas las niñas estudiadas se registraron la historia clínica (incluyendo el antecedente de exposición a hormonas o drogas desde la vida intrauterina y la administración de substancias de uso tốpico), examen físico y antropometría completa. realizados por uno de los autores. El diámetro mamario se midió con calibrador (Storme), la estatura en estadiómetro Harpenden( $R$ ) y el peso con balanza Seca(R). Además se determinó la edad ósea (mediante radiografía de mano y el método de Greulich y Pyle) y se hizo ecotomografia pelviana. ambos procedimientos fueron readizados a interpretados por un mismo observador. En la ecolomografía pelviana se uso un equipo de atta resolución Aloka 650, con transductores de 5 y 7,5 Mhz y en ella se midieron los diámetros 
longitudinal y anteroposterior del útero y el volumen ovúrico, empleanilo la fórmula "volumen ovárico= (largo $x$ ancho $\mathrm{x}$ alto)/2", modificada de Sample ${ }^{2}$. Como grupo control para los exámenes de inágenes se eligió a 39 niñas de $20,41 \pm 7,96$ meses de edad, que no tenian tejido mamario palpahle y debían realizarse ecografía abdominal por infección urinaria o dolor abdominal.

Las mediciones de LH, FSH, prolactina y estradiol en el plasma se realizaron por métodos de radioinrounnensayo. En todas las pacientes con telarquia prematura -no así en los controles-, se reajizo además una prueba de estimu10, inyectando $100 \mu \mathrm{g}$ del factor liberador de gonadotropinas (LHRH, Elen), tomando muestras de sangre para medir FSH y LH basales y $30,60,90$ y 120 minutos despucs. Asimismo se Jeterminaron niveles plasmáticos de zearalenona y dietilbestrol: separándolos previamente mediante croinatogratía en placa fina (CPF). La actividad estrogénica total en el plasma se midió a través dẹl método de ensayo de radiorteceptor (RRA) ${ }^{3}$

El grupo control para las determinaciones hormonales estuvo constituido por 18 niñas sill telarquia. de la misına edad (2l,48 $\pm 10,33$ meses) y nivel socioeconómico. elegidas al acar entre nif̂́as hospitalizadas para cirugía electiva, que debian someterse a extracción de sangre para exámenes preoperatorios. En cinco pacientes con Ielarcjuia prematura, en quienes cventualmente desaparecio el brote manrario, sc midió ntuevamente actividad estrogénica total en plasma.

Se estudió además la presencia de dierilbestrol. zerealenona (toxina de efecto estrogénico producida por un hongo que puede desarroltarse en el maiz), y actividad estrogenica total (RRA) en plasma y tejidos de pollo. Para ello se revisaron pollos de 30-35 días de edad (machos y hembras Broiler) obtenidas directamente de distintos pro. dectores avicolas del país. Estos se mantuvieron por 24 horas en vivero, alimentados con su formula habitual y posteriormente fueton sacrificados por decapitacion. Las muestras de sangre $(12 \mathrm{ml}$ ) fueron recolectadas en tubos heparinizados, a partir de las cuales se asló el plasma utilizado en los análisis posteriores. De las mismas aves se obtuvo muestras de tejido ( $100 \mathrm{~g}$ de músculo y de grasa). Además se analizaron muestras de aves provenientes de mósculo, grasa y distintos órganos, adquiridas en diferentes supermercados de la Región Metropolitana. La medición de homonas en los tejidos se realizó de acuerdo al método de Verbeke modificado ${ }^{4}$.

En el análisis estadístico se emplé́ la prueba de Student, considerando significativo un valor $p<0,05$, excepto en la comparación de la actividad estrogénica en el plasma de las niñas en las que desapareció la 1elarquia. donde se aplicó la prucba de Wilcoxon para muestras pareadas.

\section{Resultados}

Los niveles basales de LH, FSH, prolactina y estradiol no mostraron diferencias significativas entrc las pacientes con telarquia prematura $y$ las nifáas control (tabla 1). Asimismo, la relación LH/FSH basal fue similar en las niñas con o sin telarquia prematura $(0,37 \pm 0,23$ vs. $0,38 \pm$ 0,87 ). La respuesta al estímulo con L.HRH fue predominantemente de FSH, con escasa variación de LH. Las relaciones basal y máxima LH/ FSH en pacientes con telarquia prematura fueron inferiores a 0,4 (tabla 2), siendo sospechosa de pubertad precoz una relación superior a $0,66^{\circ}$. La actividad estrogénica total expresada como pg equivalentes de estradiol, fue significativamente mayor en las pacientes con telarquia prematura, con respecto a los controles, existiendo, sin embargo, sobreposición de valores en dos casos. En cinco pacientes con telarquia prematura, en cuyo seguimiento se constató regresión de la telarquia, la medición subsiguiente de actividad estrogénica dio resultados significativamente menores que la inicial y muy parecidos a los de las niñas controles (tabla 3).

El tamaño del útero no mostró diferencias entre pacientes con telarquia prematura y controles. El volumen medio de los ovarios fue mayor en pacientes con telarquia prematura, pero la diferencia no fue significativa. No se detectaron quistes ováricos o folículos mayores a 0,8 $\mathrm{cm}$ de diámetro (tabla 4).

La actividad estrogénica en tejidos de pollo (tabla 5) estaba dentro de los márgenes de las recomendaciones de $\mathrm{FAO}^{6}$.

\section{Tabla 1}

Cancentraciones hormonales basales en niñas con telarquia y controles

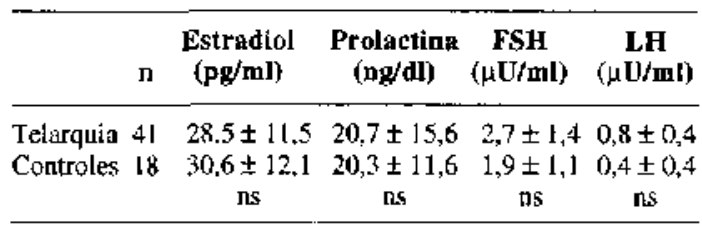

Tabla 2

Respuesta a la estimulación con LHRH en 41 niñas con telarquia prematura

\begin{tabular}{|c|c|c|c|}
\hline $\begin{array}{l}\text { Tlempo } \\
\text { (min) }\end{array}$ & $\begin{array}{c}\text { FS } \\
(\mathrm{U} \mu / \mathbf{a l})\end{array}$ & $\begin{array}{c}\text { LH } \\
(\mathbf{U} \mu / \mathbf{m l})\end{array}$ & $\begin{array}{c}\text { Relación FSH/LH } \\
(\mu \mathrm{U} / \mathrm{ml})\end{array}$ \\
\hline-15 & $2,72 \pm 1,40$ & $0.82 \pm 0.48$ & $0,37 \pm 0,23$ \\
\hline 0 & $2,40 \pm 1,25$ & $0,77 \pm 0,52$ & \\
\hline 30 & $20,11 \pm 14,02$ & $5,04 \pm 3,05$ & \\
\hline 60 & $28.74 \pm 17,59$ & $5,01 \pm 2,75$ & $0.28 \pm 0.19$ \\
\hline 90 & $28,39 \pm 18,10$ & $3,71 \pm 1,75$ & \\
\hline
\end{tabular}




\section{Tabla 3}

Actividad estrogénica total en plasma en niñas con telarquia prematura, controles y en las pacientes en que la telarquia remitió (Ex)

\begin{tabular}{lrrrr}
\hline & & \multicolumn{2}{c}{ Actividad estrogénica (RRE) } \\
& & (pg E2 equiv/mI) & Márgenes \\
\hline Telarquia & 25 & $201,12 \pm 102,37^{\star}$ & $(89-493)$ \\
Controles & 9 & $78,83 \pm 20,56$ & $(43-121)$ \\
Ex-telarquia & 5 & $75,66 \pm 8,59$ & $(62-89)$ \\
\hline
\end{tabular}

* $=p<0,01$

Tabla 4

Ecografía pelviana en niñas con y sin telarquia prematura

\begin{tabular}{|c|c|c|c|c|}
\hline & $\mathbf{n}$ & $\begin{array}{c}\text { Long. útero } \\
\text { (cm) }\end{array}$ & $\begin{array}{l}\text { Dí́m. útero } \\
\text { (ce) }\end{array}$ & $\begin{array}{l}\text { Vol. ovario } \\
\text { (cm) }\end{array}$ \\
\hline $\begin{array}{l}\text { Telarquia } \\
\text { Controles }\end{array}$ & $\begin{array}{l}41 \\
39\end{array}$ & $\begin{array}{c}2,97 \pm 0,36 \\
2,68 \pm 0,28 \\
\text { ns }\end{array}$ & $\begin{array}{c}0,64 \pm 0.14 \\
0,60 \pm 0,15 \\
\text { ns }\end{array}$ & $\begin{array}{c}0,74 \pm 0,37 \\
0.54 \pm 0,24 \\
\text { ns }\end{array}$ \\
\hline
\end{tabular}

Tabla 5

Actividad estrogénica total en 25 muestras de tejidos de pollo

\begin{tabular}{lc}
\hline & $\begin{array}{c}\text { Actividad estrogénica } \\
\text { (ng/g de tejida) }\end{array}$ \\
\hline Músculo & $0,187 \pm 0,023$ \\
Grasa & $0,295 \pm 0,043$ \\
Hígado & $0,306 \pm 0,051$ \\
\hline
\end{tabular}

\section{Comentario}

El desarrollo mamario es el primer signo puberal en las niñas y ocurre, en promedio, a los 10,9 años ${ }^{7}$. Se considera que es precoz si aparece antes de los 8 años $(-2,5 \mathrm{DE}$ del promedio), lo que plantea el diagnóstico diferencial entre pubertad precoz y telarquia aislada (la que no progresa al desarrollo puberal completo). La telarquia prematura es benigna, afecta predominantemente a niñas menores de tres años, es un motivo de consulta relativamente frecuente. No sc conoce su causa. Los mecanismos etiopatogénicos sugeridos incluyen el aumento de la concentración de estrógenos plasmáticos (E2), debido a secreción ovárica transitoria ${ }^{7}$ o administración de hormonas por vía exógena ${ }^{8-11}$; estrogenos totales normales con aumento de estrógenos libres debido a disminución de la globulina transportadora de esteroides sexuales (SBG) ${ }^{7}$; aumento de la sensibilidad del tejido mamario a concentraciones fisiologicas de estrógenos (en la mayoría de las afectadas los niveles circulantes de E2 son nornales) ${ }^{11}$; exceso fisiológico de andrógenos suprarrenales, cuya aromatizaciốn a estrógenos, podría explicar el desartollo mamario preco $z^{12}$. No ha sido posible, por otra parte, confirmar que drogas empleadas por la madre durante el embarazo (p.e. anticoncepcionales orales) estén involucrados. Entre las causas exógenas, la más discutida ha sido la contaminación de alimentos con hormonas estrogénicas. En Puerto Rico, Italia y Estados Unidos se ha encontrado indicios que sugieren que la ingestión de carne de vacuno contaminada con compuestos anabolizantes implantados en estos animales (zeranol, trembolona o compudose $(\mathrm{R}))^{12}$ o de pollo, en cuya crianza podría haberse utilizado estrógenos o substancias con acción estrogénica ${ }^{9-11}$, serían responsables de la aparición de telarquia en determinadas zonas geográficas. La zearalenona toxina con efecto estrogénico derivada del Fusarium. un hongo que puede desartollarse en los depositos de maíz, fuente habitual del alimento de las aves, es una fuente potencial de contaminación.

En la denominada "epidemia" de telarquia descrita en Puerto Rico" ${ }^{11}$ un tercio de los 482 casos estudiados tenía estrógenos plasmáticos aumentados, ovarios de mayor volumen que lo esperado y quistes ováricos. Habiéndose descartado trastornos endocrinologicos, se planteó la posibilidad de contaminación alimentaria por anabolicos, pues se encontró aumento de la actividad estrogénica en diferentes tipos de carnes analizados por estudio de receptor citosólico, si bien no fue posible identificar exactamente a la sustancia responsable. Por otra parte el dietilbestrol fue responsable del desarrollo precoz, en 7 niños (4 mujeres y 3 varones) tratados por tuberculosis en San Francisco, EUA, donde, por error, se contaminó la isoniazida con este producto. Las niñas sufrieron telarquia, pigmentacion aerolar y sangramiento vaginal, los varones ginecomastia y, en dos pacientes (un varón de 9 años y una niña de 10), se inició precozmente la 
pubertad $^{14}$. La pigmentacion areolar, constante en aquellos casos, no se registró en las niñas de nuestra serie.

En nuestro estudio no se observaron diferencias en las concentraciones plasmáticas de prolactina, FSH, LH, o estradiol entre pacientes y controles, sin embargo, la actividad estrogénica medida por RRA fue cuatro veces mayor en las niñas con telarquia prematura y descendió a valores similares que en los controles en los casos en cuyo seguimiento se comprobo desaparición posterior de la telarquia. También en ellas la respuesta de la FSH al factor liberador fue exagerada, lo que difiere de lo descrito en niñas normales y con pubertad precoz ${ }^{15,}{ }^{16}$. La respuesta de $\mathrm{LH}$ a $\mathrm{LHRH}$ en niñas con telarquia prematura es como en las normales, pero la de FSH es igual que en las que sufren de pubertad precoz, sugiriendo que en telarquia prematura habría una activación parcial del eje hipotálamo hipofisiario y gonadal que sólo afectaría a la hormona folículo estimulante ${ }^{17-19}$. Las concentraciones de FSH son, desde la primera semana de vida, mayores en las niñas que en los varones, alcanzan su máximo alrededor de los tres meses de edad, luego descienden lentamente a lo largo de los primeros dieciocho meses, alcanzando las tasas bajas y estables del período prepuberal. Otro tanto ocurre con los estrógenos plasmálicos ${ }^{18}$. La secreción nocturna de FSH aumenta en niñas con telarquia prematura, mientras en las niñas normales esto ocurre sólo inmediatamente antes de la pubertad.

Por lo expuesto anteriormente es posible postular que, durante los primeros años de vida en la mujer, babría un estado de resistencia fisiologica para una mayor actividad estrogénica FSH dependiente, que - en condiciones normalesimpediría las manifestaciones de una precocidad sexual. La telarquia prematura podría ser considerada entonces como una forma de precocidad sexual incompleta, resultante de una alteración transitoria de esta resistencia fisiológica en el eje hipotálamo, hipófisis y ovario.

La mayor respuesta de FSH y el aumento del volumen ovárico en las pacientes con telarquia prematura de esta serie, si bien no eran estadísticamente significativos, junto con el aumento de la actividad estrogénica, sugieren fuertemente activacion parcial del eje hipotalámico, hipófísiario y gonadal, predominantemente para FSH, lo que podría explicar la consecuente pro- ducción endógena, probablemente ovárica, de E2 u otra substancia no detectada por el RIA para estradiol, pero con actividad estrogénica al método de RRA. También puede postularse que una activación gonadal primaria, capaz de producir sustancias con acción estrogénica, pudiese sensibilizar el hipotálamo y la hipofisis, facilitando la secreción parcial de FSH. EI hecho que no varíen las concentraciones de estradiol en ambos grupos puede deberse a que el RIA para E2 no tiene la sensibilidad y la precisión requeridas para las concentraciones existentes en las pacientes. El RRA es un método más sensible, capaz de detectar bajas concentraciones de E2 y de otras sustancias con actividad estrogénica.

La posibilidad que substancias exógenas con actividad estrogénica fuesen responsables del desarrollo mamario, haría sospechar contaminación de alimentos habituales en los niños, pero ello parece poco probable ante la activación parcial del eje y el aumento de volumen de los oyarios y la ausencia de actividad estrogénica anómala en las muestras obtenidas de las aves de cocina. No obstante, se requeriría el análisis de mayor número de muestras y otros alimentos infantiles para obtener conclusiones más definidas sobre este punto.

La confirmación de la desaparición de la actividad estrogénica total, medida por RRA, junto con la de la telarquia, aun cuando el número de estas pacientes es bajo, es muy significativa y permite plantear una relación causa efecto entre ambas variables.

La ecografía pelviana es muy útil para estudiar la telarquia y descartar la pubertad precoz, trastorno en el cual la longitud del útero es mayor a $4,5 \mathrm{~cm}$ y el volumen ovárico supera los 2 $\mathrm{cm}$ cúbicos. Lamentablemente hay muy pocos informes que comparan los resultados de la ultrasonografía pelviana entre niñas normales y con telarquia o pubertad precoz que permitan una adecuada orientación ${ }^{15,21}$.

La ausencia de cambios en los niveles de prolactina en nuestros casos, coincide con otras series en las que, además, ella no aumentó después de estímulos con TRH, indicando que no juega ningún rol en la patogenia de la telarquia prematura ${ }^{18,22}$.

A pesar que la telarquia prematura continuó siendo, en muchos aspectos, un misterio, cuya etiopatogenia especffica no se conoce, se demuestra que en las niñas afectadas hay activa- 
ción parcial del eje hipotalámico, hipofisiario y gonadal a predominio de FSH y actividad estrogénica plasmática total aumentada, no siendo posible concluir sobre cuál de estos fenómenos ocurre en primer lugar.

\section{Resumen}

Con el propósito de estudiar las causas de la telarquia prematura (telarquia), se comparó el perfil hormonal y las características de los genitales internos mediante ultrasonografía pelviana en 41 niñas afectadas, de $21,15 \pm 9,74$ meses de edad y en 31 controles de la misma edad y nivel socioeconómico y se buscó contaminación de los alimentos con zearalenona y dietilbestrol por cromatografía en capa delgada. La actividad estrogénica total en el plasma de las niñas, así como plasma y tejidos de pollo, se midió por el método de radiorreceptor (RRA). Las concentraciones basales de $\mathrm{LH}, \mathrm{FSH}$, prolactina y estradiol eran similares en pacientes y controles. La actividad estrogénica total fue significativamente mayor en las niñas con telarquia (201 士 102 vs. $78 \pm 20 \mathrm{pg} \mathrm{E} 2$ equivalente) y descendi 6 a niveles similares que en los controles (75 \pm 8 pg E2) al desaparecer la telarquia. La respuesta a LHRH en pacientes con telarquia fue mayor para FSH $(28,7 \pm 17,5 \mathrm{U} / \mathrm{ml})$ que para $\mathrm{LH}$ $(5,01 \pm 2,7 \mathrm{U} \mu / \mathrm{ml})$. El tamaño del útero y la maduración ósea fueron similares en ambos grupos, pero el volumen ovárico medjo fue mayor en pacientes con telarquia. No se detectaron contaminantes exógenos en la sangre de Jas niñas y los tejidos de 25 pollos. La actividad estrogénica en el plasma de las aves cumplía las recomendaciones internacionales (FAO). La respuesta de FSH al factor liberador, el mayor volumen ovárico y el aumento de la actividad estrogénica sugieren activación parcial del eje hipotálamo-hipofisiario-gonodal de predominio FSH en las niñas con telarquia prematura.

(Palabras clave: telarquia prematura, estrógenos, LH, FSH, hormona liberadora, gonadorelina, contaminación de alimentos.)

\section{Referencias}

1. Wilkins S: The diagnosis and treatment of endocrine disorders in childhood and adolescence. Third edition, Springfield: Thomas, 1965: 206
2. Sample WF, Lippe B, Gypas MT: Gray scale ultrisonography of the normal female pelvis. Radiology $1977 ; 125: 477-83$.

3. Scatchard G: The attraction of proteins for small molecules and ions. Ann N Y Acad Sci 1949; 51:660672.

4. Verbeke R: Sensitive multi-residue method for detection of anabolics in urine and tissues of slaughtered animals. Journal of Chromatography 1979; 177: 69-84.

5. Oerter KE, Uniane $M$, Rose SR, Burnes $\mathrm{KM}$, Cutler $G B$ : Gonadotropin secretory dynamics during puberty in nornal girls and boys. J Clin Endocrinol Metab 1990 71: 1251-1258.

6. Ferrando R.: Alimentos tradicionales y no tradicionales. Roma. Organización de las Naciones Unidas para la Agricultura y la Alimentación (FAO), 1980.

7. Escobar ME, Rivarola C, Bergadá C: Plasma concentration of 17-B estradiol in premature thelarche and in differents types of sexual precocity. Acta Endocrinol $1976 ; 81 \div 351$.

8. Fara G, Del Carvo G, Bermuzzi $S$ et al.: Epidemic of breast enlargement in an italian school. Lancet 1979; 11: 295-297.

9. Montague-Bronm $K$ : Premature thejarche in Puerto Rico (letter). Am J Dis Child 1987; I41: 1250-1251.

10. Nizzoli $G$, Del Corvo $G$, Fara $G M$, Chiumello $G$ : Gynaecomastia and premature thelarche in a school childres population of northem Italy. Acta Endocrinol (Copenh) (Suppl) 1986; 279; 227-231.

11. Sáenz de Rodriguez CA, Bongiovanni AM, Conde de Borrego L: An epidemic of precocious development in Puerlo Rico's children. J Pediatr 1985; 107; 393-396.

12. Landier F, Chaussain $J$, Job JC: Isolated breast prematurity in young girls. Arch Fr Pediatr 983; 40: $549-552$

13. Chasalow FI, Granoff AB, Tse TF. Blethen SL: Adrenal steroid secretion in girls with pseudoprecocious puberty due to autonomous ovarian cysts. J Clin Endocrinol Metab 1986; 63: 828.834

14. Weber W, Grossmann M. Thom J, et al.: Drug coutamination with diethilbestrol. New Engl J Med 1963; 268. 411-415.

15. Stanhope $R$, Abdulwahid NA, Adams J. Brook CG: Siudies of gonadolrophitu pulsatility and pelvic ultrasound examinations distinguish between isolated premature thelarche and central precocious puberty. Eur J Pediatr 1986; 145: 190-194.

16. Pescoviz $O H$, Hench $K D$, Barnes $K M$, Loriaux $D L$. Cutier GB Jr.: Premature thelarche and central precocious puberty: the relationship between clinical presentation and the gonadotropin response to luteinizing hormone-releasing hormone. I Clin Endocrinol Metab I988; $67 ; 474-479$.

17. Pasquino AM, Tebaldi L, Cioschi L, Cives $C$, Finacchi G, Maciocci $M$. Mancuso G, Boscherini B: Premature thelarche: a follow up study of 40 girls. Natural history and endocrine findings. Arch Dis Child 1985; 60: 1180-1182.

18. Beck W, Wutke W: Diurnal variations of plasma luteinizing hormone, follicle-stimulating hormone and prolactine in boys and girls from birth to puberty. $J$ Clin Endocrinol Metab 1980; 50: 635-639. 
19. Ilicki A, Prager Lewin R. Kauli R. Kaufmun $H$. Schachier A, Laron Z: Premature thelarche natural history and sex botmone secretion in 68 girls. Acta Paediatr Scand 1984; 73 : 756-762

20. Beck W, Stubbe S: Pulsatile secretion of luteinizing hormone and sleep related gonadorropin rhytms in girls with premature thelarche. Edir J Pediatr 1984: 141: 168-170.

21. Showker TH, Comite F, Rieth $K G, D$ wyer AJ, Cutler
GB Jr, Loriaux DL: Ulurasound evaluation of female isosexual precocious puberty, J Ultrasound Med 1984; 3: 309-316.

22. Abe $K$, Massuser $N$, Nahara Y, Fujita $H$, Fujieda $K$. Kuto T. Mikami $Y$ : Prolactine response to thyrotropinreleasing hormone in children witl gynecomastia, premature thelauche and idiopathic precocious pubery. J Exp Mod 1984; 142: 283-288.

Esta publicación estâ disponible en copias de microfilms de 16 y $35 \mathrm{~mm}$ y microfichas de $105 \mathrm{~mm}$, las que pueden solicitarse a:

University Microfilms International 300 North Zeeb Road

Ann Arbor, Michigan 48106, USA.

This journal is also available in $16 \mathrm{~mm}$ microfilm, $35 \mathrm{~mm}$ microfilm and 105 mm microfilm copies through

University Microfilms Intemational, 300 North Zeeb Road,

Ann Arbor, Michigan 48106, USA. 